\title{
RECENSIONES
}

\section{Editio Hungarica}

Péter Kovács: Die Geschichte Pannoniens in der spätrömischen Zeit (284-395 n. Chr.). Pytheas Kiadó, Budapest 2019. - ISBN 978615-5741-14-2 - 304 Seiten

Zuletzt im Jahre 1975 erschien eine umfassende Monographie über das spätkaiserzeitliche Pannonien. ${ }^{1}$ Das Buch von András Mócsy besteht aus 212 Seiten, aber nur auf 77 wurde die 111 Jahre lange, von 284 bis 395 andauernde Epoche, die von Péter Kovács in seinem neuen Band auf 304 Seiten behandelt wird, erörtert. ${ }^{2}$ Es ist leicht einzusehen, dass die Veröffentlichung einer neuen Zusammenfassung schon aktuell war. Die Geschichte einer kürzeren Periode (284-363) wurde vom Verfasser im Jahre 2016 auf Englisch publiziert, aber im Vergleich dazu vertritt dieser Band eine erweiterte Ausgabe, die an die ungarischen Leser, in erster Linie an die Fachleute und Studenten, gerichtet ist. ${ }^{3}$ P. Kovács nahm in der vorliegenden Arbeit nicht auf sich, aufgrund der manchmal lückenhaften Quellen die Geschichte fließend zu erzählen: Der Verfasser konzentrierte sich äußerst auf die Quellen und er analysierte die zur Verfügung stehenden Texte. Mehrere, bis dahin nur wenig oder gar nicht bekannte Quellen treten im Buch auf, das ohne die ebenfalls von P. Kovács gezeichneten sieben Bände der Quellenveröffentlichungs- und Übersetzungsreihe Fontes Pannoniae Antiquae nicht entstanden sein könnte. Es ist besonders nützlich, wenn der Leser die Bände 6-7 bei der Hand hat, da der Verfasser am Ende der einzelnen Kapitel alle in den Kapiteln angewandten Quellen in einem Appendix erwähnt. Das geschieht aber aus Platzmangel in der ursprünglichen Sprache, also können die in den Fontes lesbaren Übersetzungen für die im Altgriechischen oder Latenischen nicht bewanderten Leser eine Hilfe leisten - aber nur bis 337, weil die Übersetzung der Quellen von 338 bis 395 auch da fehlt.

Der Hauptteil des Buches besteht aus sechs, im Großen und Ganzen chronologischen Kapiteln von der Tetrarchie bis dem Tod des

${ }^{1}$ A. Mócsy: Pannónia a késői császárkorban [Pannonien in der späten Kaiserzeit]. Akadémiai Kiadó, Budapest 1975.

${ }^{2}$ In einer neulich veröffentlichten Zusammenfassung wurde dieser Zeitabschnitt etwa auf 67 Seiten vorgestellt: M. SZABÓ-L. BORHY: Magyarország története az ókorban: kelták és rómaiak [Die Ge-

DOI: $10.1556 / 072.2019 .70 .2 .8$
Iulianus Apostata. Das einzige Kapitel, das in die chronologische Ordnung nicht hineinpasst, ist das „Martyres Pannoniae“, das über die überraschend viele pannonische christliche Märtyrer ein umfassendes Bild bietet. Es ist ein Redaktionsproblem, dass die Geschichte der Christen der gegebenen Zeit auch in den chronologischen Kapiteln die in dem der Märtyrer Gelesenen wiederholend - auftaucht. Vier Anhänge schließen den Band, nämlich ein über SEG 31, 1116 (Ein phrygischer Soldat in Pannonien), ein über den Ortsnamen Constantia in der Provinz Valeria, ein über die in Beremend gefundenen Inschrift des Valerius Dalmatius und ein anderer über die pannonischen kaiserlichen Sitze. Um die Zusammensetzung der einzelnen chronologischen Kapitel erfassen zu können, nehmen wir das erste Kapitel, in dem die Zeit der Tetrarchie in Pannonien vorgestellt wird. Dieses Kapitel besteht aus den folgenden Unterkapiteln: Die Schlacht von Margum. Wo verstarb Carinus? Die Ansiedlung der Karpen in Pannonien; Die Consularia Constantinopolitana und die Gegenfestungen; Galerius und der lacus Pelso: Die Einleitung des Plattensees in die Donau. In jedem Unterkapitel wurden alle zur Verfügung stehenden inschriftlichen oder literarischen Quellen (unter ihnen auch die Stempelziegel und die Münzen) - manchmal auch tabellarisch - vorgestellt, sogar in der ursprünglichen Sprache zitiert.

In einem solchen, an Materialien reichen Band blieben zwangsläufig kleinere und größere Tippfehler und eigenartige Satzbildungen, sogar erwies sich auch die ungarische Übersetzung der aus der englischen Ausgabe übernommenen Tabellen nicht immer als erfolgreich. Z. B. blieb der Titelkopf der zweiten Tabelle auf der Seite 276 englisch (Date; Place; Source) und auf der Seite 277 bewahrte der Name des Gratianus die englische Form (Gratian) in der ersten Tabelle.

Im folgenden Satz blieb ein Wort vermutlich aus (S. 154): ,... da auch die sich darauf beziehenden ... können fiktiv sein.“

schichte Ungarns in der Antike: Kelten und Römer]. L'Harmattan, Budapest 2015.

${ }^{3}$ P. KovÁcs: A History of Pannonia in the Late Roman Period I (284-363). Antiquitas I/67. Dr. Rudolph Habelt GmbH, Bonn 2016.

Acta Archaeologica Academiae Scientiarum Hungaricae 70 (2019) 397-402 0001-5210 (C) 2019 Akadémiai Kiadó, Budapest 
Auf der Seite 213 ist der Satz in Verbindung mit Aginatus nur schwer zu verstehen. Von der Aufzählung der kleineren Tippfehler und Inkonsequenzen wird diesmal abgesehen.

Trotz des fragmentarischen, inselartigen Aufbaus gelang es, im behandelten Band das zumeist stürmische Jahrhundert der Geschichte der Provinz vorzustellen, als die Struktur der Provinz radikal verändert und die Bevölkerung ausgetauscht wurde. Trotz der kleineren Schönheitsfehler und der nicht unbedingt leserfreundlichen Struktur werden dieser Band und die geplante Fortsetzung als Ausgangspunkt aller späteren Pannonien-Forschungen dienen. Diese Arbeit und die Bände der Reihe Fontes vertreten eine Leistung, die bestimmt, wie man die spätkaiserzeitliche Geschichte Pannoniens in der Zukunft verfasst. Im Vorwort stellt es sich weiters heraus, dass der Verfasser schon an einer ebenfalls lückenfüllenden Zusammenfassung, an der Vorbereitung von Fontes Hunnorum arbeitet: Im Letzteren werden die Quellen der europäischen Hunnen angehäuft. Dabei wird natürlich auch Pannonien eine bestimmende Rolle spielen.

György Németh

Institut für Alte Geschichte der Eötvös-Loránd-Universität 6-8. Múzeum krt., H-1088 Budapest, Ungarn nemeth.gyorgy@btk.elte.hu

\section{Editiones externae}

Holger Komnick: Die Fundmünzen der römischen Zeit aus dem Bereich der Colonia Ulpia Traiana. Xantener Berichte 29. Darmstadt 2015. - ISBN 978-3-8053-4972-7 - 606 Seiten, 49 Tabellen, 17 Tafeln

Im Jahre 2015 erschien die Aufnahme und Bearbeitung der römischen Fundmünzen aus dem Bereich der Colonia Ulpia Traiana (CUT). Obwohl das Projekt „Fundmünzen der römischen Zeit in Deutschland“ (FMRD) vor mehr als einem Jahrzehnt eingestellt wurde, gibt es zum Glück noch Arbeiten, welche sich mit den Fundmünzen einiger römischer Orte bzw. Gebiete befassen. Zu diesen gehört das Werk von Holger Komnick, in dem der Verfasser in einem auf drei Jahre angelegten Projekt, gefördert mit DFG-Mitteln und durch den Landschaftsverband Rheinland, die Fundmünzen aus dem Bereich der Colonia Ulpia Traiana im heutigen Xanten bearbeitete. Seine Arbeit wurde von Martin Müller in die Xantener Berichte aufgenommen und in einem mehr als 600 Seiten dicken Buch herausgegeben.

Die Colonia Ulpia Traiana wurde von Kaiser Traianus gegründet und gehörte zu den größten Städten nördlich der Alpen. Das Gebiet war schon unter Drusus durch die Römer besiedelt worden und hier entstand eine wichtige Siedlung, die auch als Handelsplatz bis zum Bataveraufstand existierte. Die aufständischen Bataver haben sie niedergebrannt, aber wegen der günstigen Lage wurde die Siedlung wieder errichtet und sogar erweitert. Allerdings wurde sie wegen der Erhebung zu Colonia durch Traian abgerissen und einplaniert. Auf dem Gebiet wurde dann eine neue Stadt aufgebaut, die 275 n. Chr. beim Einfall der Franken zerstört wurde. Erst um 310 wurde eine neue Siedlung auf den neun zentralen Insulae errichtet. Als Lager der Legio XXX Ulpia Victrix wurde sie mit Mauern umgeben. Obwohl sie 352 von den Franken eingenommen wurde, diente sie ab 359 n. Chr. wieder als Stützpunkt der Römer. In der ersten Hälfte des 5. Jahrhunderts wurde die Siedlung endgültig verlassen. Von der Bedeutung der Colonia Ulpia Traiana zeugen neben den schriftlichen Quellen die reichen archäologischen Funde wie auch die Münzen.

Das Buch besteht aus zwei Teilen. In einem Katalog werden die Fundmünzen - sowohl Einzelfunde als auch Schatz- und Grabfunde -

${ }^{1}$ St. V. Pighius: Hercules Prodicius 1. Editiones Neolatinae 347. Wien 2010, 64. Nachdruck der Ausgabe von 1587.

${ }^{2}$ Codex Berolinensis 139. lat. qu. 36: Roma Traiana, sive Notitia Romanarum Antiquitatum, quae praecipue ex Coloniae Traianae et Veterum Castrorum <am Rande: ubi nunc est oppidu Sanctese> ruderibus, tam ab adfluente Rheno, quam a fossoribus quandoque sunt detectae et extractae. In qua nonnulla exhibentur Romana numismata, nach Fundkomplexen dargelegt. Im anderen Teil werden die Münzen nach den Epochen der einzelnen Kaiser in Hinsicht auf den Münzverkehr analysiert.

Am Anfang des Buchs legt der Verfasser eine detaillierte Forschungsgeschichte dar. Die erste Erwähnung von Fundmünzen aus Xanten findet sich in dem erstmals 1587 erschienenen Buch Hercules Prodicius des Xantener Kanonikers Stephanus Vinandus Pighius (1520-1604). ${ }^{1}$ Die ersten bestimmbaren Fundmünzen finden sich jedoch in dem 1643 begonnenen Codex Berolinensis 139 des Hermann Ewich (1601-1673). ${ }^{2}$ Obwohl Ewich über 200 Fundmünzen in recht genauen Zeichnungen dokumentierte, liegt lediglich im Fall von zwei Münzen der explizite Vermerk vor, dass sie aus dem Bereich der Colonia stammen (Nr. 3798 und 4400). Wir können nur bedauern, dass der Verfasser keine Zeichnung dieser Münzen aus dem Codex veröffentlicht, obwohl eine der beiden, der Solidus des Kaiser Honorius (Mediolanum, RIC 1206), zu den jüngsten bekannten Münzen aus Colonia Ulpia Traiana gehört. ${ }^{3}$

Die schon als archäologisch zu bezeichnende Erforschung der archäologischen Denkmäler begann nach der Gründung des Niederrheinischen Altertumsvereins im Jahre 1877, dessen Grabungsberichte in den Bonner Jahrbüchern erschienen. Eine systematische Ausgrabungstätigkeit erfolgte aber in CUT erst ab dem Ende der 1950er Jahre. Obwohl das numismatische Material von Xanten schon seit dieser Zeit gesammelt und aufgenommen wurde, entstand im Rahmen des FMRD-Projektes kein diesbezüglicher Band. Holger Komnick konnte diese Materialsammlungen neben den archivalischen und Literaturquellen in seinen Katalog eingliedern. Der Katalog folgt dem FMRD-Gliederungssystem, jedoch hat der Verfasser die archäologischen Angaben (Fundort, Fundumstände, Literatur usw.) fast zu jeder Münze aufgenommen und im Anmerkungsteil hinter dem Katalog gebracht (S. 273-402). So können die Münzen eine wichtige Hilfe für die zeitliche Bestimmung der Schnitte und archäologischen Erscheinungen sein. Nach den Einzelfunden kommen die in den Gräbern gefundenen Münzen (S. 403-409), dann die Schatzfunde (S. 409-462). Für die Archäologen ist die Konkordanz der Schnitte mit den Münzen besonders hilfreich, weil sie eine bessere Übersichtlichkeit ermöglicht (S. 466-504). In den Gräbern kamen hauptsächlich römische Bronze-

marmora, signa, sigilla et supellectilia, diverso, prout oblata sunt, tempore, observata, depicta et collecta ab Hermanno Ewichio Vesaliense, antehac in Sanctensi nunc Vesaliensi reformata Ecclesia ministro (Vesaliae. Anno M DC XLIII. Mense Ianuario).

${ }^{3}$ Ebenda, fol. 37. Im Ewichs Codex ist bei dieser Münze Folgendes notiert: „Hic aureus nummus Honorii dicitur inventus esse in ruderibus Coloniae Traianae" (s. Komnick S. 395). 
münzen zum Vorschein. Unter den fast ein Dutzend Schatz- und Kollektivfunden gibt es einige sehr interessante - wie zum Beispiel der innerhalb eines Hauses am kleinen Hafentor im Juli 1977 gefundene Goldschatz, der aus 16 Aurei von Tiberius bis Vespasianus bestand (S. 410-412), oder der Schatzfund aus der Insula 20 aus dem Jahr 1964, der neben 274 Denaren und 116 Antoninianen auch Gold- und Silberringe, Silberlöffel, goldene Ohrringe, ein Silberschälchen und Bruchsilber enthielt (S. 423-445). Der Schatz wurde während der Regierungszeit von Postumus verborgen.

Im zweiten Teil des Buchs bringt der Verfasser eine erste Auswertung der antiken Fundmünzen aus dem Bereich der Colonia Ulpia Traiana, wobei sein Katalog als Quelle ausgewertet wurde. Die im Katalog aufgenommenen 4601 Einzelfunde und fast 1000 Münzen aus Gräbern und Schatz- bzw. Kollektivfunden boten die Grundlage zur Untersuchung des Münzverkehrs von Colonia Ulpia Traiana. Sein Vorhaben ist der Auswertung der Carnuntiner Münz- und Schatzfunde ähnlich, die jedoch von einer Forschergruppe durchgeführt wurde. ${ }^{4}$ Der Verfasser hat zum Vergleich mit der CUT-Einzelfundmünzreihe weitere Fundreihen aus dem Raum der Provinz Germania inferior herangezogen. Er analysiert die Fundmünzen nach Kaisern und ab 284 nach Prägeperioden.

Aus dem Bereich der CUT sind nur drei griechische Bronzemünzen bekannt, was jedoch kein Einzelfall ist, wie die vom Verfasser zitierten Funde bezeugen. Die 40 keltischen Münzen sind dem römischen Geldumlauf zuzurechnen. Die 57 republikanischen Münzen könnten, wie der Verfasser hinweist, nach langer Umlaufzeit erst im
1. Jahrhundert $n$. Chr. hier erschienen sein. Die Prägungen von Augustus sind in besonders hoher Zahl (815 Stück) vertreten und von den 799 Bronzemünzen der augusteischen Zeit sind 174 als Barbarisierungen eingestuft (S. 522). Besonders interessant ist, dass aus dem Bereich der so wichtigen Colonia Ulpia Traiana nur so wenige Goldmünzen bekannt sind. Neben dem Schatzfund mit 16 Aurei sind das je ein Aureus des Claudius I. (Nr. 1371, Rom, RIC2 25) und des Traianus (Nr. 2517, Rom, RIC 4) sowie ein Solidus des Honorius (Nr. 4400, Mediolanum, RIC 1206), welche die besondere Rolle der Goldmünzen im römischen Münzverkehr zeigen. ${ }^{5}$ Holger Komnick beschäftigt sich ausführlich mit den Schatz- und Kollektivfunden (S. 559-577) und diskutiert, ob sie Teile eines Fundhorizontes sind, wobei er zahlreiche schriftliche, numismatische und archäologische Angaben nutzte. Schon bei dem Aureischatz aus dem Bereich des Hauses am kleinen Hafentor in der CUT zeigt der Verfasser, wie vorsichtig man mit den Verbergungsgründen umgehen sollte (S. 562-563).

Das Buch von Holger Komnick ist eine sehr wichtige Arbeit sowohl für die numismatische und archäologische Forschung wie auch für die Wirtschaftsgeschichte. Es zeigt auch die Möglichkeiten und die Bedeutung der Grundlagenforschung, die so oft vernachlässigt wird.

Péter Prohászka Archeologický ústav SAV v Nitre 2., Akademická, 94921 Nitra, Slovakia prohaszkapeter@freemail.hu
${ }^{4}$ M. Alram-Fr. Schmidt-Dick (Hrsg.): Numismata Carnuntina. I-III.: Forschungen und Material. DAWW 353. Wien 2007.
${ }^{5}$ Siehe zur Problematik der Goldmünzen zuletzt: S. F. PFAHL: Goldmünzen des 1.-5. Jahrhunderts zwischen Rhein und vorderem Limes. Provinzialrömische Studien 4. Weinstadt 2013. 
Corpus der römischen Funde im europäischen Barbaricum. Polen. 3.: Ireneusz Jakubczyk: Mittelpolen. Kraków 2018. - ISBN 978-83-7676-303-3 - 204 Seiten, 104 Tafeln.

Das europäische Forschungsvorhaben der Aufnahme und Veröffentlichung der römischen Funde im Barbaricum (CRFB) hat neue Anstöße bekommen. Nach den CRFB Deutschland-Bänden sind in kurzer Zeit zwei auch in Polen erschienen. Das Forschungsvorhaben CRFB fing in Polen mit der Aufnahme der römischen Funde in Masuren an. ${ }^{1}$ Dann erfolgte eine Pause von fast zwei Jahrzehnten, bis der Band Kleinpolen von Piotr Kaczanowski bearbeitet und nach seinem tragischen Tod von Judyta Rodzińska-Nowak im Jahre 2017 herausgegeben wurde. ${ }^{2}$ Kaum ein Jahr später ist wieder ein Band erschienen, in dem die römischen Funde aus Mittelpolen bearbeitet wurden.

Gegenüber den anderen Materialaufnahmen, wie zum Beispiel in Deutschland, wo die Arbeit nach Bundesländern aufgeteilt wurde, ${ }^{3}$ erfolgte die Bearbeitung in Polen nach Woiwodschaften. Im neuen Band wurde aber beim geographischen Rahmen ein anderes Konzept verwendet, wobei der Begriff „Mittelpolen“ eine wichtige Rolle spielt. Wie der Autor erläutert (S. 9): „Obwohl der Begriff Mittelpolen oft in der Fachliteratur vorkommt, war dessen Gebiet verschiedenartig definiert... Dabei ist es wichtig zu sagen, dass Mittelpolen weder im geographischen noch historischen Sinn ein begrenztes Land ist noch sich mit den Verwaltungsgrenzen deckt. Es ist vielmehr als archäologischer Fachausdruck zu verstehen." Etwas später bestimmt der Autor jenes Gebiet, das im Band bearbeitet wurde (S. 9): ,Im Rahmen des Bandes wird Mittelpolen als Gebiet zwischen Warthe, Prosna, Bzura und Pilica definiert. Administrativ gehört der Großteil des Gebietes zur Woiwodschaft Łódź, deren Umfang zugleich die Ost-, Süd- und Nordostgrenze des Forschungsareals markiert. Die Nordgrenze verläuft entlang des Ner-Flussgebietes und der Warthe bis zu ihrem Nebenfluss Prosna." Aus diesem Gebiet sind im Katalog einige vorrömische Funde aus dem Ausgang der jüngeren vorrömischen Eisenzeit in der Zeit des Augustus berücksichtigt worden. Die jüngsten Funde sind an den Übergang vom 5. zum 6. Jahrhundert n. Chr. datiert. Wie der Autor hinweist (S. 11), liegt das Arbeitsgebiet im Bereich der Przeworsk-Kultur, weswegen das chronologische System von H.-J. Eggers und K. Godlowski angewandt wurde, während für die frühe und ältere Kaiserzeit das System von T. Liana genutzt wurde. ${ }^{4}$

Mittelpolen wird als Gebiet mit besonders zahlreichen Importfunden betrachtet. Der Autor bringt eine ausführliche Forschungsgeschichte (S. 12-16) des Gebietes. Der erste bekannte römische Fund kam im Jahre 1854 ans Tageslicht (S. 12). Im Dorf Dębe wurde ein Brandgrab mit einer Bronzekasserolle E 151 und Fragmenten einer gerippten Glasschale E 181 entdeckt (S. 68). Eine detaillierte Beschreibung mit Fotos veröffentlichte erst B. Podczaszyński im Jahre 1893. ${ }^{5}$ Die erste Veröffentlichung der Kasserolle (Abb. 1) erfolgte jedoch schon im Jahre 1859 in der Mitteilung der k. k. Zentralkommission in Wien, was dem Verfasser unbekannt war. ${ }^{6}$ Die Beigaben des

${ }^{1}$ Corpus der römischen Funde im europäischen Barbaricum. Polen. 1.: Masuren. Hrsg.: W. NowaKowski. Warszawa 2001.

${ }^{2}$ Corpus der römischen Funde im europäischen Barbaricum. Polen. 2.: P. KaCZANOwsKi: Kleinpolen. Kraków 2017.

${ }^{3}$ Siehe zum Beispiel: Corpus der römischen Funde im europäischen Barbaricum. Deutschland. 4.: M. ERDRICH: Hansestadt Bremen und Bundesland Niedersachsen. Bonn 2002.

${ }^{4}$ T. LianA: Chronologia względna kultury przeworskiej we wczesnym okresie rzymskim [Relative Chronologie der PrzeworskKultur in der frühen Römerzeit]. WiA 35 (1970) 429-471.

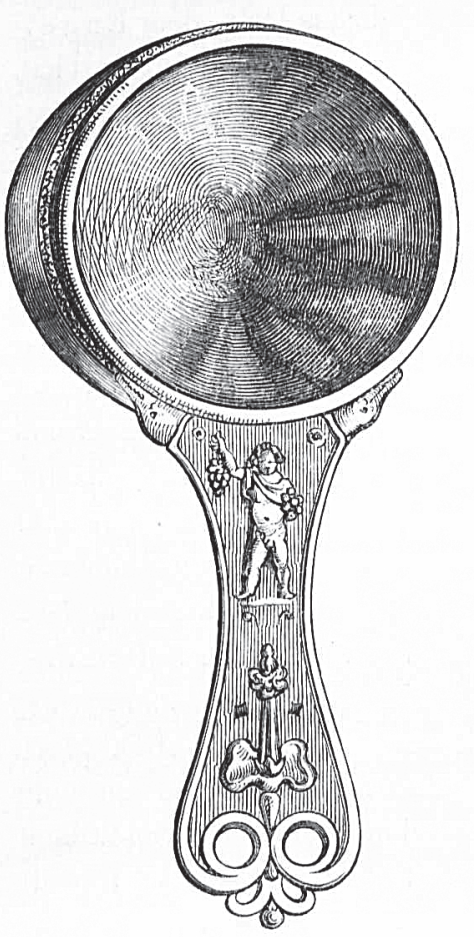

Brandgrabes wurden von der gelehrten Gesellschaft in Krakau mit zahlreichen anderen archäologischen und kunsthistorischen Gegenständen im September 1858 im fürstlichen Lubomirski'schen Palais ausgestellt. Über die Fundumstände hat R. von Eitelberg Folgendes berichtet: „Wir geben hier die Abbildung eines antiken Gefässes, das in dem zwei Meilen von Kalnisch entfernten Dorfe Domba in Russisch-Polen gefunden worden sein soll. Es hat am Griffe einen bachischen Genius mit Weintrauben in dem Bilde der römischen Zeit, ein ausserordentlich schönes Ornament am Rande, das sich in Monumenten ähnlicher Art und auch an Bauwerken, als Spinen und Toren vorfindet. Das Gefäss hat eine prächtige Patina.“

Jakubczyk unterscheidet drei Perioden in der Forschungsgeschichte. Die erste reicht bis zum Jahre 1918, die zweite von 1919 bis 1945 und die dritte ist die Zeit ab 1946. Besonders viele Importstücke brachte die zweite Periode, was seiner Meinung nach mit der Entstehung der Institutionen - wie zum Beispiel des Städtischen Museums in Łódź im Jahre 1918 - im Zusammenhang stand. Besonders interessant ist seine Behauptung, dass sich trotz der Intensivierung von Ausgrabungen im Bereich der zukünftigen Autobahnen und anderer Unternehmungen von 1985 an die Zahl entdeckter Importe aus Mittelpolen als wesentlich schwächer erweist (S. 14).

${ }^{5}$ B. PodCZASZYŃSKi: Wykopalisko z grobu cialopalnego we wsi Dębe pod Kaliszem [Die Ausgrabung eines Brandgrabes im Dorf Dębe bei Kalisz]. Zbiór Wiadomości do Antropologii Krajowej 17 (1893) 47-50, Taf. I.1-5.

${ }^{6}$ R. vON EITELBERG: Die archäologische Ausstellung der gelehrten Gesellschaft in Krakau. Mitteilungen der k. k. Central-Commission zur Erforschung und Erhaltung der Baudenkmale 4 (1854) 34-35. 
Besonders wichtig ist das Kapitel „Die Forschungsgeschichte der Elitengräber in Mittelpolen“ (S. 17-21), weil Jakubczyk hier die Fund- bzw. Forschungsgeschichte der Elitengräber, darunter derjenigen von Łęg Piekarski, zu rekonstruieren versucht. Er nutzt dabei neben den literarischen Quellen auch die Archivalien. In Łęg Piekarski wurden 1933 und 1936 besonders reiche Gräber entdeckt, die in der Fachliteratur als klassische Beispiele der Lübsow-Gräber (Elitengräber der älteren Kaiserzeit) gelten. Die frühesten Zufallsfunde aus den Jahren 1849-1860 sind über hundert Jahre später als Fürstengrab A in die Literatur eingegangen. In den 1930er Jahren wurden die ersten Funde im März 1933 registriert, als beim Ausgraben „zahlreiche Bronzeurnen" und andere Gegenstände gefunden wurden. W. Antoniewicz hat dann die Funde angekauft. Drei Jahre später kam während der Ackerarbeiten ein Steinpflaster von $5 \times 2,5 \mathrm{~m}$ mit der Grabkammer darunter zum Vorschein. Nach einer Untersuchung befanden sich im Besitz des Entdeckers zwei Silbergefäße, zwei Bronzeschüsseln, eine Bronzekanne, ein Kleinmesser und eine Schere aus Bronze sowie gläserne Spielsteine und Spielwürfel. Z. Durczewski hat die Fundstelle archäologisch untersucht. Dabei zeigte es sich, dass Fußboden und Wände der Grabkammer aus Holzbalken gebaut worden waren. Im unberührten Grabteil fand er in situ zwei Bronzeeimer E 24 und E $37-$ 39. Wie der Autor erwähnt, erwecken die Informationen zu Grab III die größten Zweifel. Nach dem Bericht von L. Leciejewicz wurden mehrere Brandgräber während der Erdarbeiten im Jahre 1947 entdeckt und in einem davon befanden sich mehrere metallene Beigaben. Nach einem anderen Bericht kamen die Beigaben in einer Grabkammer aus Feldsteinen von 1,8 $\times 1,2 \mathrm{~m}$ ans Tageslicht. Die hier gefundenen Bronzegefäße (Eimer E 39-40, Kasserolle E 141, Kelle und Sieb E 162) und zwei Silbergefäße (E 170) zeugen vom Reichtum des Grabes. Wegen der einander widersprechenden Berichte werden besonders die Angaben über die Grabform bezweifelt. Die erste Kontrollgrabung erfolgte erst in den Jahren 1975-1977, wobei auch das zwischen 1849 und 1860 während des Brunnenbaus entdeckte „Fürstengrab“ lokalisiert wurde. Damals wurde der Südteil der Grabkammer beschädigt. Im Nordteil haben sich Fragmente von zwei Silberskyphoi und eine Fibel A IV 75 in situ erhalten. Der Verfasser beschäftigt sich anhand der Archivalien auch mit der Fundgeschichte des „Fürstengrabes“ 22 in Witaszewice (S. 20-21).

Hier müssen wir aber bemerken, dass es besser gewesen wäre, wenn die Beigaben der Elitengräber auf eigenen Tafeln abgebildet worden wären. Der Verfasser gliedert den Tafelteil nach Materialgruppen: Auf die Silber- und Bronzegefäße folgen die Gläser, Terra Sigillaten und römische Tongefäße, Fibeln, Perlen und am Ende die Münzen. So hätte er das in der Einleitung bestimmte Ziel besser erreicht (S. 7): ,Die Fundumstände der am reichsten ausgestatteten Gräber werden hier detailliert betrachtet, da sie oft nicht genügend hervorgehoben wurden. Diese Angaben sind für die Interpretation und die Diskussion über die Geschlossenheit der Grabkomplexe äußerst wichtig." Im Tafelteil sind die Funde entweder zeichnerisch oder fotografisch abgebildet.

In dem Kapitel „Bemerkungen zum Katalog“ deutet der Verfasser darauf hin, dass im Katalog die römischen Importfunde nach dem System der Reihe CRFB aufgenommen wurden. Hier finden wir auch kurze Analysen einzelner Fundgruppen (S. 22-25).

${ }^{7}$ A. ALFÖLDI: Anyaggyüjtés a római pénzek Magyarországon készült egykorú utánzatainak osztályozásához. III.: Római aranymedaillonok utánzatai mint germán nyakékek - Materialien zur Klassifizierung der gleichzeitigen Nachahmungen von römischen
Der Katalogteil umfasst mehr als 100 Seiten (S. 55-157) und gliedert sich alphabetisch nach den Fundorten. Bei den einzelnen Funden hat Jakubczyk sowohl die Funde aus den Museen bzw. verschiedener Sammlungen als auch Literatur und Archivalien eingearbeitet. Neben der Bronzekasserolle E 151 von Dębe möchten wir noch auf einen Fund hinweisen, bei dem wichtige Literaturangaben vom Verfasser nicht verwendet wurden. Der reichste Hortfund von Mittelpolen kam im Herbst 1926 oder im Frühling 1927 in Zagorzyn ans Tageslicht (S. 152-153). Die Bronze- und Goldgegenstände, mindestens 3000 römische Denare aus dem 1.-2. Jahrhundert und fast zwei Dutzend Goldmünzen aus dem 4. Jahrhundert lagen in einem Bronzebecken. Der Hortfund wurde größtenteils an Sammler, Vermittler und Händler verkauft, daher ist eine vollständige Rekonstruktion der $\mathrm{Zu}$ sammensetzung nicht mehr möglich. Ein Teil wurde zerstört oder eingeschmolzen. Noch dazu befanden sich die erhaltenen Funde bis 1945 in Museen in Berlin, Kalisz, Kiel, Warszawa, Wrocław, Stuttgart und New York. In seiner wichtigen Arbeit über die Nachahmungen römischer Münzen beschäftigte sich Andreas Alföldi auch mit dem Medaillon von Valentinianus I. (Taf. 93.1). ${ }^{7}$ Diese Angabe war dem Verfasser unbekannt. Alföldi schrieb aber auch etwas über die Fundgeschichte: „Fund von Nedzierzewo bei Kalisch. Er ist leider nach allen Windrichtungen zerstreut worden. Das Breslauer Museum rettete davon eine Riemenschnalle aus massivem Silber, einen Goldanhänger und Solidi von Valentinian II. (so richtig!), Gratian und Theodosius. Ein großes Goldmedaillon von etwa $750 \mathrm{Gr}$. Gewicht soll eingeschmolzen worden sein, ein anderes, unediertes Goldmedaillon von Valentinian I., das durch Dr. R. Gaettens-Halle Sept. 1927 erworben worden ist und dann nach Amerika (Slg. Newell) wanderte, scheint auch aus diesem Funde zu stammen. Er erwarb gleichzeitig auch ein kleineres Multiplum von Constantinus II. (Mm. 26, Gr. 8.97), das durch den Handel weiterging. Dr. Gaettens verdanken wir den Gipsabguss des ersteren, bisher unbekannten Stückes Taf. IIIa.10." Nach Alföldi befand sich also im Hortfund eine Riemenschnalle aus massivem Silber und ein großes Goldmedaillon von ca. $750 \mathrm{~g}$ wurde eingeschmolzen. Das Goldmedaillon im Münzkabinett Berlin, eine wohl barbarische Nachahmung von Valens (?), hat auch ein solches Gewicht (S. 152). Die Medaillons von Valentinianus I. und Constantin II. wurden verkauft und gelangten zuerst in die Sammlung Newell, dann der American Numismatic Society. Wir können nur hoffen, dass vielleicht noch irgendwelche Angaben über die Gegenstände und auch zur Fundgeschichte in den Archiven auftauchen.

Am Ende des Textteils befinden sich die Listen (Fundplätze, Schlüsselnummern, Fundtypen usw.). Die erhaltenen Funde sind auf 114 Tafeln abgebildet. Der Band schließt mit einer Faltkarte, worauf sich die nummerierten Fundorte befinden.

Der dritte Band der CRFB Polen ist eine wichtige und willkommene Arbeit zur Erforschung der römisch-barbarischen Kontakte in Mitteleuropa. Wir können nur hoffen, dass auch andere Gebiete ebenfalls in Bearbeitung sind.

Péter Prohászka Archeologický ústav SAV v Nitre 2, Akademická, 94921 Nitra, Slovakia prohaszkapeter@freemail.hu

Münzen aus Ungarn und den Nachbarnländern. III.: Nachahmungen römischer Goldmedaillons als germanischer Halsschmuck. NK 28-29 (1929-1930) [1933] 12 und Taf. IIIa.10. 